\title{
Calles para todos. repensando las calles
}

\section{Streets for everyone. rethinking the streets}

DOI: 10.179 81/mod.arq.cuc.18.1.2017.05

Fecha de Envío: 25 de agosto de 2017 Fecha de Aceptación: 27 de octubre de 2017

\author{
Manuel Alberto Londoño Cárdenas \\ Más Urbano (https://www.masurbano.org/) \\ masurbanocolombia@gmail.com \\ Ana Carolina Restrepo Acosta \\ Más Urbano (https://www.masurbano.org/) \\ arestrepo805@gmail.com

\section{Laura Elena Zuluaga Fernandez \\ Más Urbano (https://www.masurbano.org/)} \\ lauzuluf@gmail.com
}

Para citar este artículo:

Londoño, M., Restrepo, A. y Zuluaga, L. (2017). Calles para todos. Repensando las calles. MODULO ARQUITECTURA-CUC, vol. 18, no. 1, pp. 91-108. DOI: 10.17981/mod arq cuc 18.1.2017.05

\section{Resumen}

Siempre, las calles de nuestras ciudades se han concebido como lugare de circulación, de intercambio y de encuentro. Pero con el paso de tiempo, especialmente con la llegada de los automóviles y la planeación del movimiento moderno, las calles se han considerado principalmente como sendas de circulación rápida donde muchas veces la inseguridad nos excluye. Las calles se han vuelto, en muchas ciudades, lugares hostiles donde prima el motor y no los pasos, donde las máquinas a una velocidad exagerada ocupan un espacio predominante dejando rezagado a los caminantes a un espacio claustrofóbico e ínfimo. Y aunque para los años sesenta o setenta diversos colectivos, profesionales y autores (Buchanan, Mumford, Jacobs, entre otros) promovían un enfoque menos técnico-simplificador y más completo en las calles a partir de visiones distintas ${ }^{1}$, nuestras ciudades aún tienen un alto problema de cohesión, de seguridad, de contaminación y de integración social. Es por esto que hoy en día buscamos repensar las calle y las llamamos "calles completas", dándoles un adjetivo integral para recordar que estas deben ser diseñadas y construidas para facilitar el acceso seguro a todas las personas, independiente del medio de transporte que decidan usar, sin importar su edad o condiciones especiales de movilidad (Smart Growth America, 2018). Estas deben permitir el acceso a los predios privados y, sobretodo, brindar conexiones y estancias seguras entre los lugares de vivienda y de trabajo, estudio u ocio. En pocas palabras, las calles deberían ser el lugar central por excelencia de nuestro espacio público; lugares polivalentes que se transforman para abarcar a todos y recibir diferentes actividades con la misma evolución y diversidad de nuestra cultura

Palabras clave: Movilidad urbana, sostenibilidad, espacio público, accesisibilidad universal, calles completas.

\section{Abstract}

It seems like always the streets in our cities have been conceived as places of circulation, exchange and meeting. But with time, especially with the arrival of automobiles and the planning of the modern mobility, streets have been mainly considered as paths of rapid circulation where insecurity often excludes us. They have become, in many cities, hostile places where engines prevail and not steps, where machines at an exaggerated speed occupy a predominant space leaving behind walkers to a claustrophobic and insignificant space.

And although by the sixties or seventies various groups, professionals and authors (Buchanan, Mumford, Jacobs, among others) promoted a less technical and simplistic approach of streets to give way to a more complete approach that included different perspectives, our cities still have a high problem of cohesion, security, pollution and social integration. That is why today we seek to rethink the streets and call them "complete streets", granting them a comprehensive adjective to remember that these must be designed and constructed to facilitate safe access to all people regardless of the means of transport they choose to use or regardless of the age or special mobility conditions (Smart Growth America, 2018). In sum, streets should allow access to private property and, above all, provide connections and safe spaces between people's homes and workplaces, or schools and leisure spaces. In short, the streets should be the central place par excellence of our public space; polyvalent places that are transformed to embrace everyone and receive different activities with the same evolution and diversity of our culture.

Keywords: Urban Mobility, sustainability, public space, universal accesibility, complete streets.

1 Del mismo modo, para la época se comenzó a introducir en Europa expresiones como: "convivir con el tráfico", "calles de coexistencia" y "ciudad peatonal" como implicación institucional para disminuir los problemas viales, de tráfico y para mejorar la convivencia en la ciudad Herce y Francesc, 2002a). 


\section{HistoRIA DE LA CALLE}

Desde la antigüedad, en ciudades como Babilonia o Atenas, los trazados de las calles respondían principalmente a la necesidad de conexión entre templos o lugares sagrados. Eran calles donde los recorridos se convertían en ceremonia y estaban cargadas de diversos elementos simbólicos de la sociedad. Vías amplias y bellamente decoradas como la Vía Procesional de Marduk o vías de cortejo como la Vía Sagrada que cruzaba el Ágora (AA.VV, 2014). Luego, Roma como la primera gran ciudad de la historia donde, a excepción de algunas vías importantes de conexión con la región, sobresalían las calles que se extendían siguiendo un crecimiento desordenado y orgánico.

En la Edad Media, con el auge de las rutas comerciales, los viajes y el intercambio entre mercaderes y artesanos, la calle se convirtió en un espacio sin orden, atiborrada de usos, lo que llevó a la degradación y a la insalubridad de estos espacios urbanos. Sin embargo, con la llegada del Renacimiento, la ciudad comienza a ajustarse; la burguesía pasa a controlar la ciudad y se empieza a organizar la calle para hacerla más útil, estética e higiénica. Así, la calle se vuelve un lugar donde se privilegian las perspectivas para distinguir los edificios principales o lugares exclusivos para pasear y contemplar.

La ciudad industrial se bate entre el desarrollo económico y la transformación social, convirtiendo la calle en un lugar de rutinas, polución y, sobre todo, en soportes de las nuevas invenciones del transporte público. Es así como, por un lado, el Plan Haussmann para París subordinaba todas las funciones del asentamiento urbano a la propia calle (Camarasa, 2015). Calles amplias y rectilíneas que se abrían paso sobre pequeñas calles para transformar no solo el espacio sino también la relación pública. Por otro, el proyecto de reforma y ensanche para Barcelona planteado por Cerdá, formulaba la transformación de la calle, no solo bajo el concepto de circulación o de ventilación, sino también bajo la idea de 'entidad conjuntiva' entre la vía pública y los edificios que la limitan lateralmente. Proponía la calle como el complemento perfecto e indispensable de cada vivienda y del mismo vecindario ya que brindan luz, aire, vistas y, sobre todo, dan la posibilidad de la sociabilidad y que, además, dan paso a todos los servicios, al transporte y a los diferentes transeúntes, respondiendo así al organismo social y urbano (Cerdá y Soria i Puig, 1996).

Años más tarde, el movimiento moderno niega la calle basado en que estas eran insalubres y ruidosas. Precisamente, se sectorizan los espacios y se ubican los edificios independientes de las calles. Estas se jerarquizan según la lógica de los trayectos en autos y se organizaban en función del tráfico y la velocidad, presentando el sistema de las $7 \mathrm{~V}$ o velocidades. $Y$ aunque en la propuesta teórica cada vía cumplía una función en una red conjunta, este sistema de reglas fue dando una respuesta parcial donde la alta consideración del tráfico afectó gravemente la coherencia urbana (Busquets, 2004) (Portas, 2007). De esta forma, en las ciudades del s. XX se comienza a hablar de las "vías segregantes" y aún hoy en día encontramos estas "calles incompletas". 
En la actualidad, el concepto que se abre paso lentamente es el de la sostenibilidad. El urbanismo contemporáneo, enfocado desde un proyecto urbano integral, apunta a la creación de espacios colectivos como objeto de recualificación, complemento y extensión de infraestructuras, identidad social, equipamiento y medio ambiente, donde las características principales en la transformación de las calles deben ser: la articulación, la flexibilidad, la revitalización o la innovación, beneficiando así el contexto donde se interviene. En otras palabras, darle a esta estructura compleja, la calle, un carácter aglutinador y de articulación con vocación de centralidad para permitir que la prolongación de la calle vuelva a ser la plaza y no el parqueadero.

\section{Diseño de la calle}

En los últimos tiempos, la movilidad en las ciudades se regula y se jerarquiza principalmente en relación a "los ingenios" que nos facilitan el movimiento y no a nosotros mismos. En otras palabras, las proporciones de las calles y su funcionamiento han sido delegadas al tamaño y al comportamiento de los carros bajo las directrices del desplazamiento rápido, olvidándonos del ser humano y su relación con sus receptores de distancia (oído, vista y olfato) y el desplazamiento lento (Gehl, 2009).

Es por esto que es necesario que se deba insistir en el estudio de esas dimensiones humanas, no solo desde una mirada ergonómica sino también desde la sociología y la antropología, ya que la especialización de la calle afecta directamente el riesgo del encuentro, la diferencia y la misma heterogeneidad de esta, un elemento primordial en el espacio público. Hay que pensar en el espacio que ocupamos, pero también en el espacio que necesitamos, primariamente, como peatones.

Justamente, "la arrogancia del espacio" es el concepto usado por Mikael ColvilleAndersen (2014), experto en movilidad urbana, para nombrar la desigual distribución de espacios públicos. De una manera muy didáctica, este autor nos plantea un ejercicio bastante lúdico para entender cómo le hemos dado más espacio a una minoría que se mueve dentro de una máquina, que a la verdadera vida de las ciudades y al desplazamiento de la mayoría a través de la movilidad activa: el caminar, el uso de la bicicleta, etc.

Como ejemplo de esta metodología, se presenta, a continuación, la representación de una esquina en la ciudad de Barranquilla, la carrera 38 con calle 38 . En amarillo, el espacio privado o semiprivado, en rojo, el espacio dedicado a los vehículos motorizados, y en azul, el espacio dedicado a los peatones, que, en algunos casos, está invadido también por los vehículos (mal parqueo).

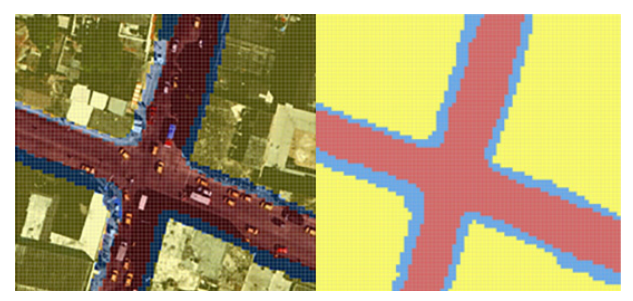

Fig. 1. Representación de la distribución del espacio en la carrera 38 con calle 38 , Barranquilla.

Fuente: elaboración propia. 
Es así, como se ve claramente, que el espacio no construido se da casi por completo al tránsito vehicular y muy poco para el tránsito de peatones, y casi inexistente para el tránsito de bicicletas. Aunque se podría asumir que las bicicletas podrían moverse por la calzada, en realidad esto se vuelve peligroso e incómodo, ya que nunca se pensó en ellas en el diseño, lo que hace que mucha gente se abstenga de hacerlo.

Muertos en incidentes viales

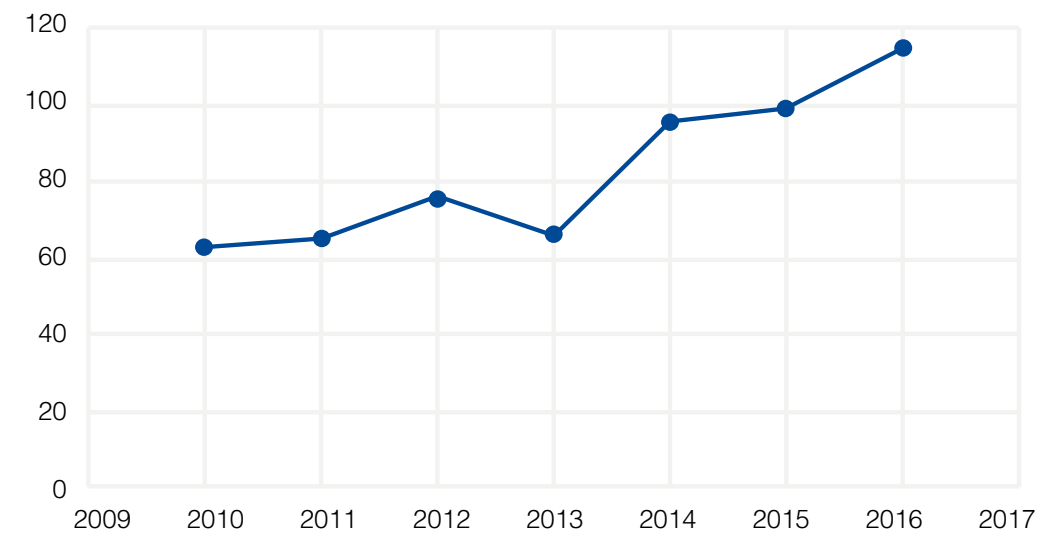

Heridos en incidentes viales

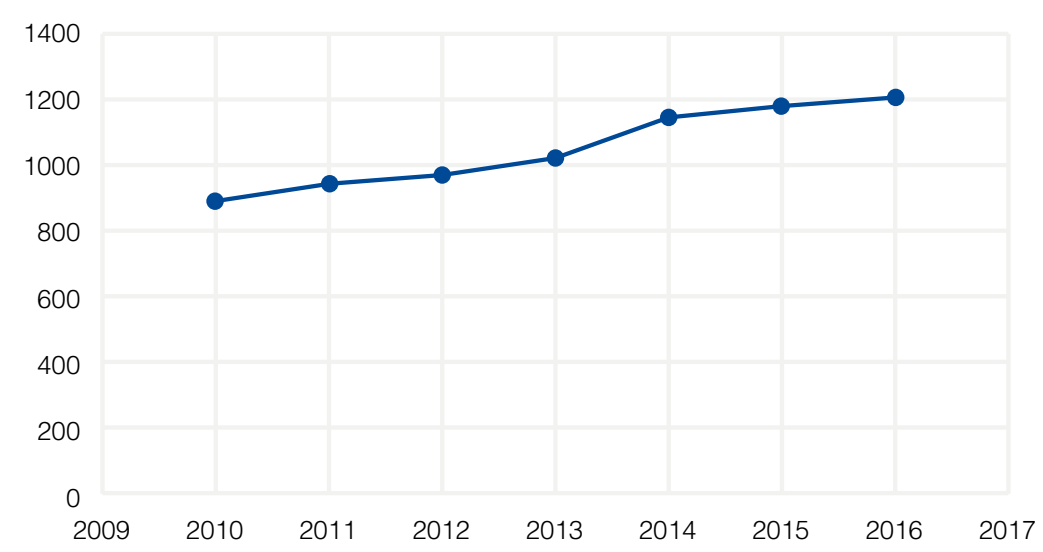

Fig. 2. Muertes y heridos en incidentes de viales en Barranquilla, 2015.

Fuente: gráfico propio con datos del Instituto Nacional de Medicina Legal y Ciencias Forenses (2017). 
Esta inseguridad en las calles no solo se basa en una sensación de riesgo e incertidumbre con respecto al tráfico; esta se ve justamente argumentada en las estadísticas de accidentes en vías que cada año está en aumento, como se puede observar en la Fig. 2.

Solo en Barranquilla, en 2015, hubo 100 muertos y 1209 heridos causados por accidentes de tránsito. Con un aumento de la población, según proyecciones del Departamento Administrativo Nacional de Estadística-Dane², donde

2 Población Barranquilla en 2017: 1'228.271; proyección 2020: 1'239.518. Tomado del Dane (2005-2020). los niveles de contaminación acústica y de polución son bastantes altos y la baja calidad ambiental se ve reflejada en un incipiente $0,92 \mathrm{~m}^{2} / \mathrm{hab}$ de espacio público $\left(1.433 .993 \mathrm{~m}^{2}\right)$, esta ciudad, como muchas otras en Colombia, debe comenzar a plantear nuevos modelos y diseños adecuados en la definición de espacios urbanos que integren y presenten calles más seguras, más accesibles y más fáciles de usar para todos.

En el proceso, se crean mejores lugares como objeto de recalificación, complemento o extensión de infraestructuras, equipamientos y medio

Usuarios de la calle

\section{Peatones}
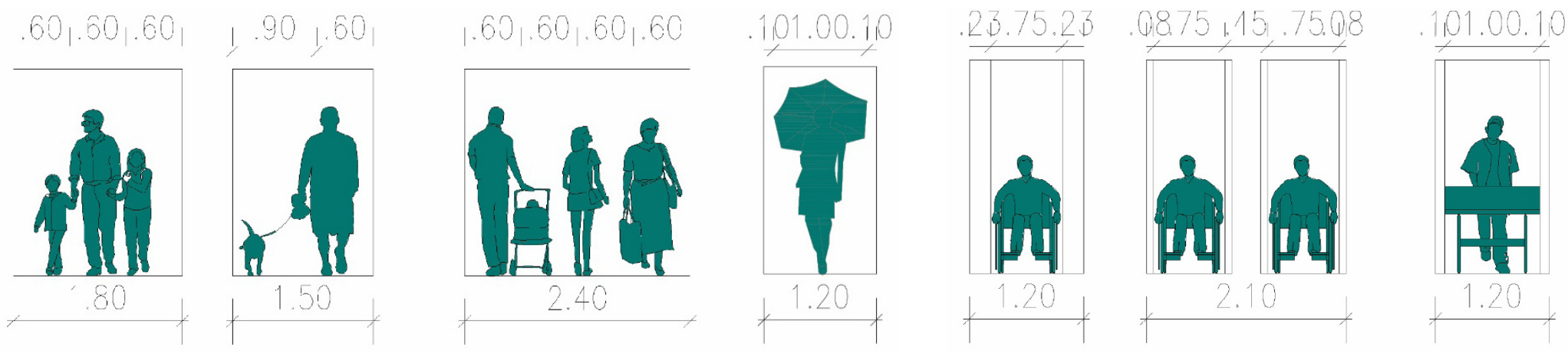

Fig. 3. Usuarios de la calle: peatones. 
Ciclistas
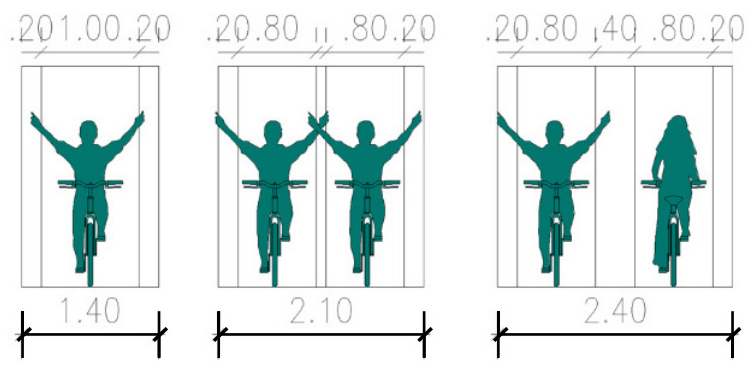

Fig. 4. Usuarios de la calle: ciclistas.

Fuente: elaboración propia.

ambiente, para que las personas vivan, jueguen y trabajen: una calle completa (Smart Growth America, 2015). Es decir, hacer de la calle una infraestructura fundamental que no se impone como traza invariable que anula la identidad de los fragmentos y, en cambio, añade otros fragmentos de otra escala y dimensión que le brindan un carácter aglutinador y de articulación con voca-

\section{Vehículos \\ motorizados \\ (particulares)}

\section{0}
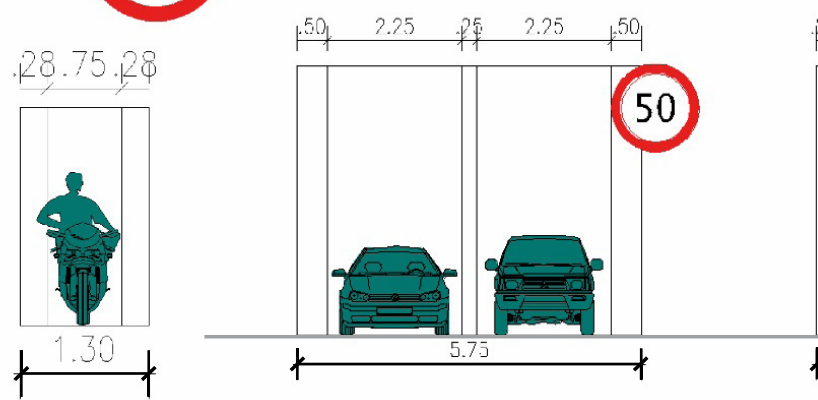

Fig. 5a. Usuarios de la calle: vehículos motorizados (particulares).

Fuente: elaboración propia.

ción de centralidad (Herce y Francesc, 2002b).

Además, entender la calle en relación con el contexto que la delimita de una manera consistente y adecuada se vuelve importante para la regeneración de los sitios que habían perdido su potencia de viabilidad y significación. Es por esto que la importancia de su diseño no debe solo primar en su traza sino 
Vehículos

motorizados

Transporte

(particulares)

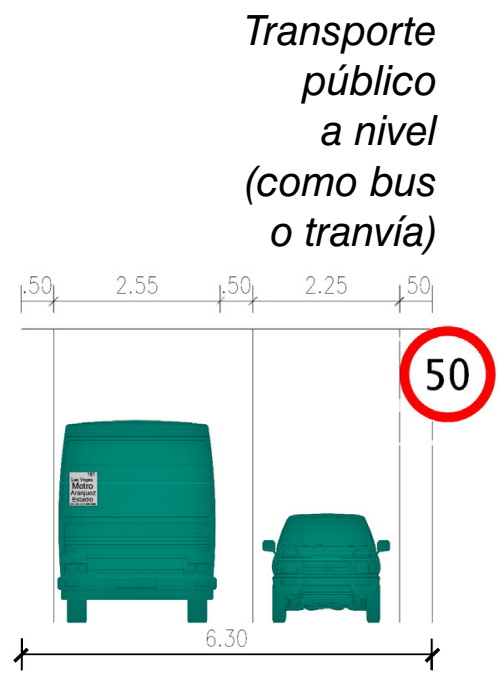

Fig. 5b. Usuarios de la calle: vehículos motorizados (particulares).

Fuente: elaboración propia.
50

Fig. 6. Usuarios de la calle: transporte público a nivel.

Fuente: elaboración propia. también en su composición ambiental (mobiliario, arborización, espacios adyacentes de complemento, etc.) como escenario propicio para el encuentro.

De esta manera, un buen diseño de calle debe comenzar con el reconocimiento integral de la circulación y estancia de todos los usuarios de esta, teniendo al peatón como beneficiario principal.
Por otro lado, un buen estudio y análisis de los componentes de la calle, según los diseños heredados y las realidades vigentes de nuestras ciudades, brindan también la posibilidad de transformar o crear nuevos espacios de una manera más sostenible. A continuación, un pequeño inventario de algunos de esos elementos que actualmente definen nuestras calles. 


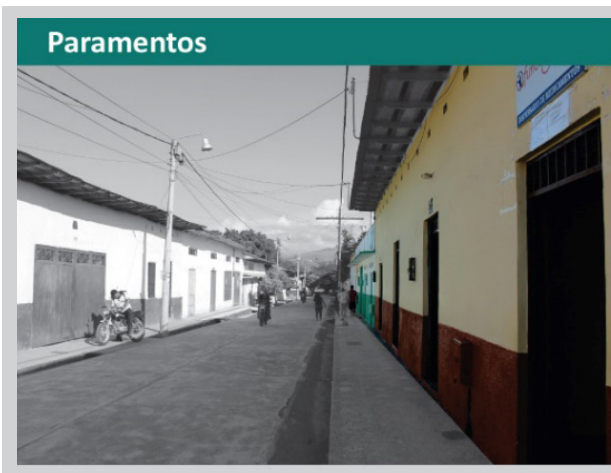

La calle está limitada por los paramentos, estos incluso hacen parte de la calle como elemento contenedor $y$ calificador.

Fig. 7. Componentes de la calle: paramento.

Fuente: elaboración propia.

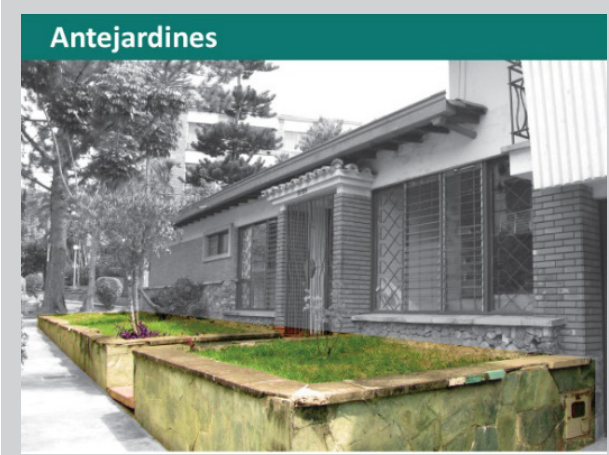

Los antejardines son los espacios intermedios entre la construcción y la calle. En Colombia estos son espacios privados de uso público.

Fig. 8. Componentes de la calle: antejardín.

Fuente: elaboración propia.

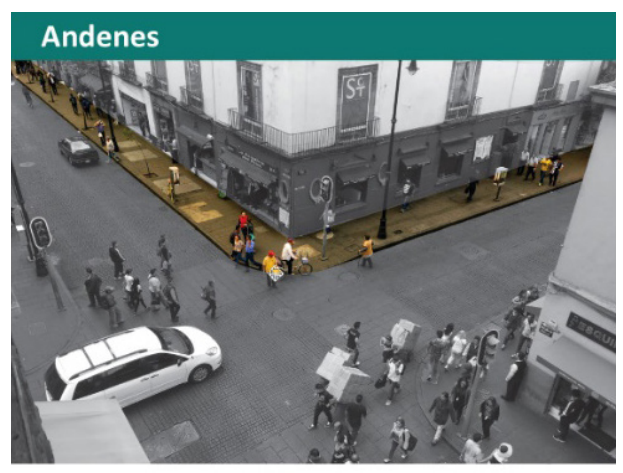

Los andenes son las superficies a cada lado de la calle, para el movimiento y encuentro de peatones o para otras actividades sociales.

Fig. 9. Componentes de la calle: andenes.

Fuente: elaboración propia.

\section{Franja de circulación}

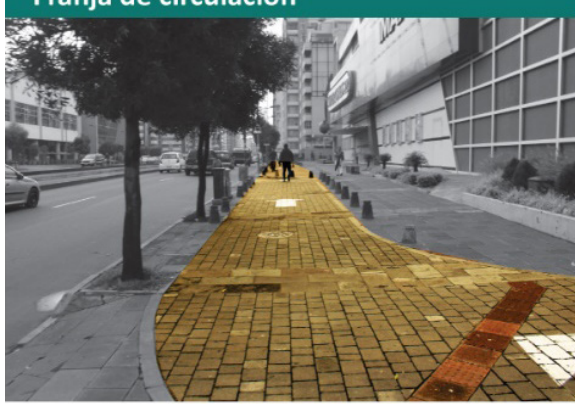

La franja de circulación es el espacio destinado para que los peatones puedan desplazarse de un lugar a otro. Su ancho debe ser proporcional a la cantidad de personas que transitan en esta.

Fig. 10. Componentes de la calle: franja de circulación. Fuente: elaboración propia.

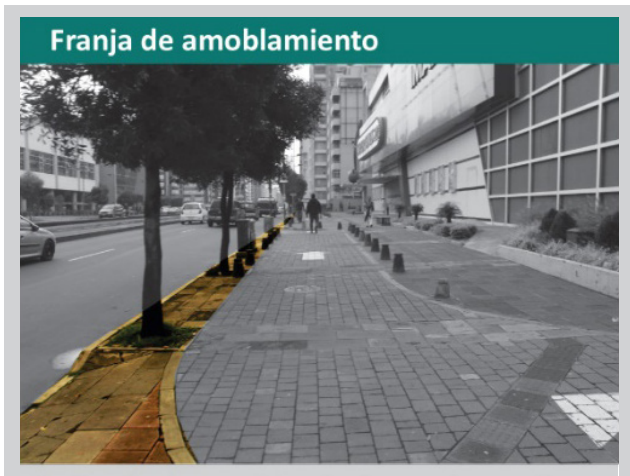

La franja de amoblamiento es el espacio dedicado para ubicar los elementos urbanos necesarios y de complemento para el buen funcionamiento de la calle.

Fig. 11. Componentes de la calle: franja de amoblamiento.

Fuente: elaboración propia.

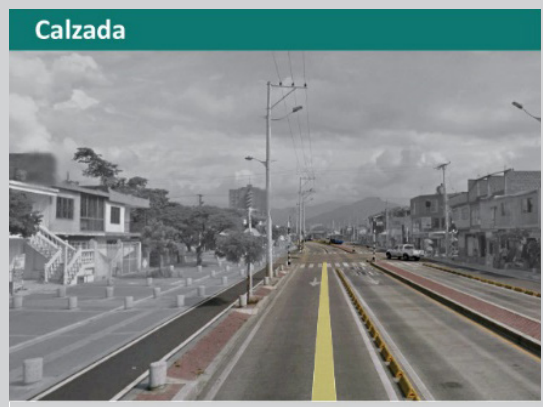

La calzada es la parte de la calle destinada al tránsito de los vehículos. Esta compuesta por carriles y pueden tener lugares para estacionar o para detenerse. Antes de la aparición de los carros era simplemente una calle ancha o calle principal.

Fig. 12. Componentes de la calle: calzada.

Fuente: elaboración propia. 


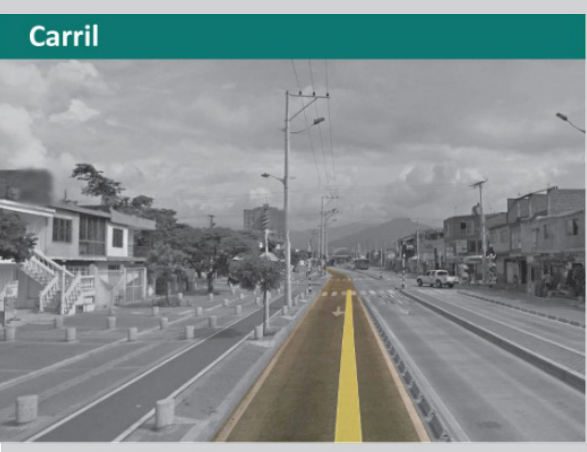

El carril es una franja longitudinal delimitada para el tránsito de vehículos.

Fig. 13. Componentes de la calle: carril.

Fuente: elaboración propia.

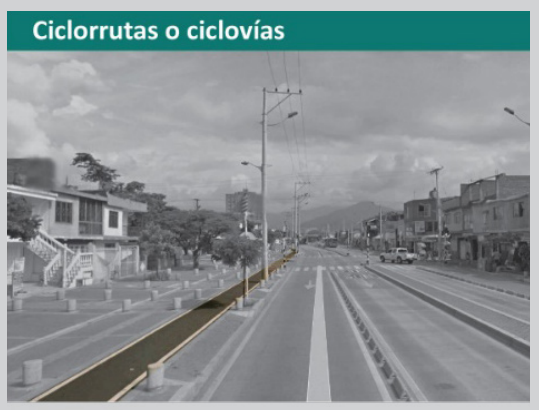

Las ciclovías o ciclorrutas son carriles exclusivos para el tránsito de bicicletas. En Colombia se acepta que por estas se muevan otros medios no motorizados.

Fig. 14. Componentes de la calle: ciclorruta o ciclovía. Fuente: elaboración propia.

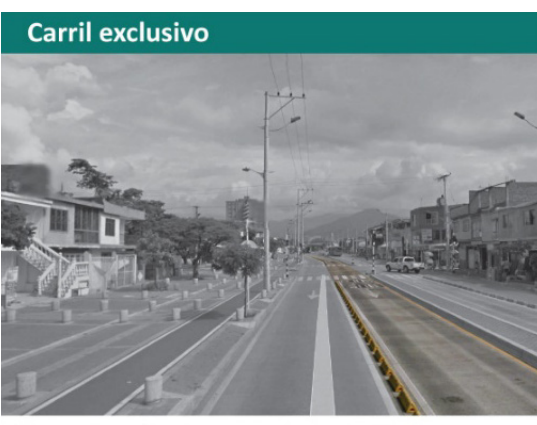

El carril exclusivo o preferente de transporte público es el espacio destinado para los buses de alta frecuencia u otro tipo de transporte masivo de personas.

Fig. 15. Componentes de la calle: carril exclusivo.

Fuente: elaboración propia.

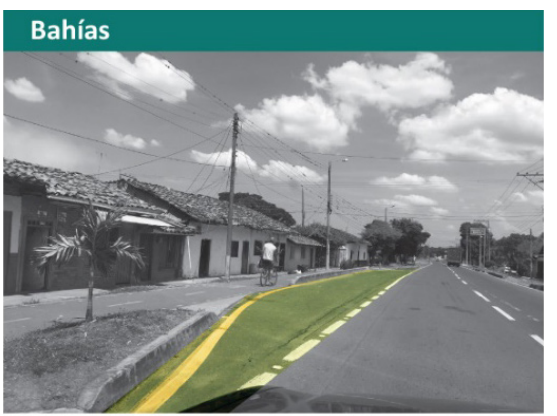

Las bahías son espacios entre la calzada y el andén destinadas para las paradas de los vehículos. Pueden ser para transporte público o privado.

Fig. 16. Componentes de la calle: bahías.

Fuente: elaboración propia.

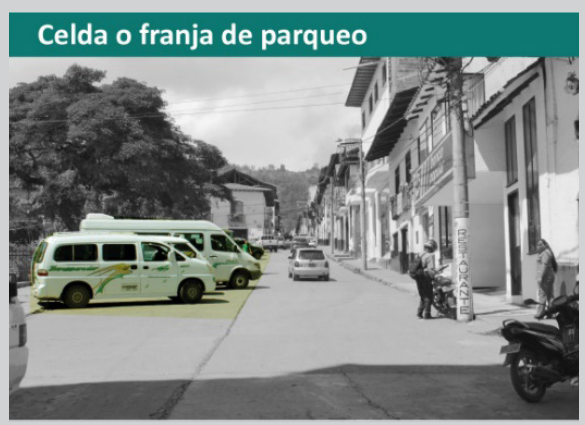

Espacio delimitado para el estacionamiento de vehiculos por un tiempo restringido (celdas con parquímetro) o indefinidamente.

Fig. 17. Componentes de la calle: celda o franja de parqueo.

Fuente: elaboración propia.

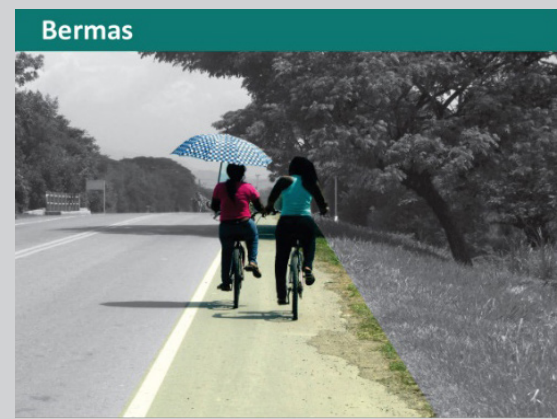

Las bermas son el espacio que existe al lado derecho de las calzadas y que permite el movimiento de todo tipo de medios y el estacionamiento transitorio.

Fig. 18. Componentes de la calle: berma.

Fuente: elaboración propia 


\section{Separador}

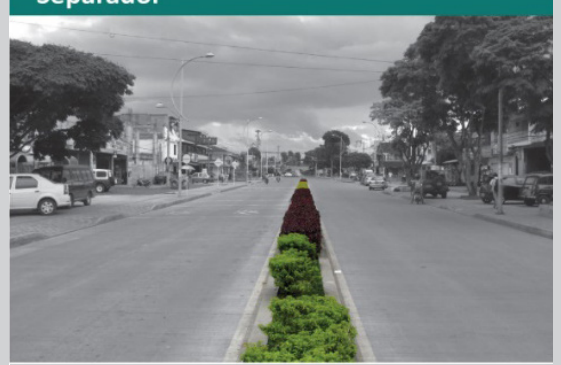

El separador es la faja que independiza los carriles. Estos pueden ser físicos (con elementos naturales o artificiales) o virtuales, es decir, señalados solo con pintura.

Fig. 19. Componentes de la calle: separador.

Fuente: elaboración propia.

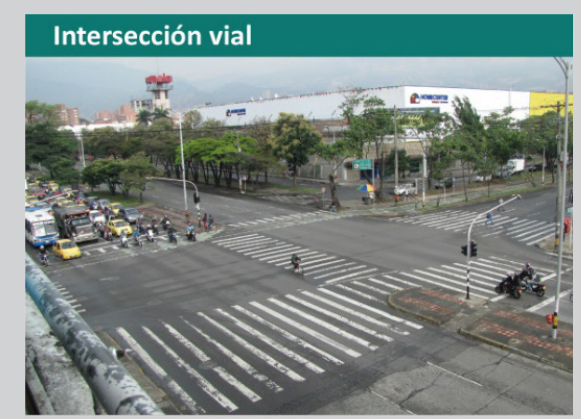

La intersección vial es el lugar donde se encuentran dos o más calles y permite el cambio de dirección.

Fig. 20. Componentes de la calle: intersección vial. Fuente: elaboración propia.
Por último, la distribución modal que se presente en el transformado sistema de calles y de movilidad debe permitir la real posibilidad de escoger entre los distintos modos y presentar las mismas condiciones, según la necesidad de cada usuario (una distribución equilibrada). Asimismo, se debe plantear que el espacio de enlace que se presenta entre los modos de circulación no sean sitios residuales, y en cambio, sean lugares agradables en el plano físico, psicológico y social; un diseño de calidad que dé la sensación de sentido del lugar animando a la gente a estar allí (Gehl, 2009).

\section{La bicicleta como instrumento de transformación urbana}

El caso de la Avenida San Juan en Medellín, Colombia. El papel de la bicicleta va más allá de darles solo la oportunidad a las personas de bajos recursos de poder moverse en la ciudad o de mejorar la salud física y mental de quien la usa. Las ciudades deben apostarle a la bicicleta ya que es el vehículo que más personas puede movilizar a muy bajo costo y a una buena velocidad $y$, aunque es cierto que su competitividad se ve mermada cuando se superan los $5 \mathrm{~km}$, las cifras en el área metropolitana del valle del Aburrá, donde uno de los principales origines y destinos es Medellín, nos indican que la distancia media que las personas caminan, por ejemplo, es de 1,75 km, y 
que, más o menos, el $27 \%$ lo emplean como su medio principal para llegar a su destino (Área Métropolitana del Valle del Aburrá, 2017). Estas cifras podrían ser un incentivo para seguir insistiendo en la implementación real de la bicicleta como opción de integración con otros medios, y así ampliar la cobertura del transporte público. Además, la infraestructura para las bicicletas es más limpia, agradable y segura para los peatones que, hasta en algunos casos, puede llegar a ser compartida con estos.

Precisamente, para conocer las virtudes de promover el uso de la bicicleta en nuestras ciudades, presentamos un caso de estudio en una de las principales intersecciones de Medellín: la Calle San Juan (vía estructurante de ingreso del occidente al centro de la ciudad) y la Carrera 65 (vía arteria que atraviesa la ciudad de sur a norte).

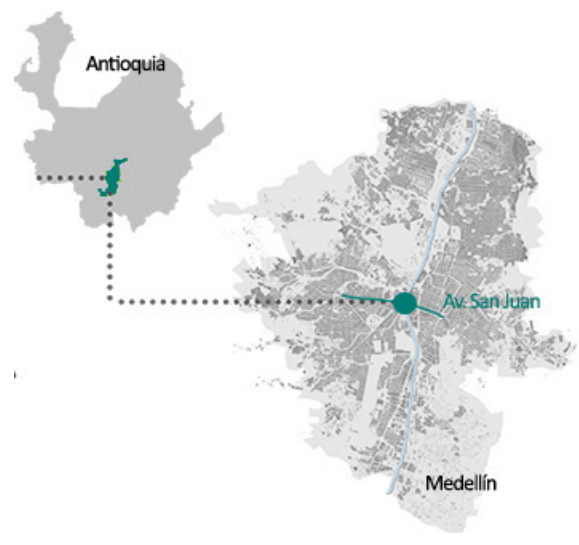

Fig. 21. Imagen de localización Avenida San Juan, Medellín.

Fuente: elaboración propia.
Actualmente, la vía tiene dos ciclorrutas bidireccionales: una que va de sur a norte y de norte a sur, y otra que va de oriente a occidente y de occidente a oriente; sin embargo, esto no siempre fue así.

Para el año 2014, solo existía la ciclorruta sobre la carrera 65, que va de norte a sur y de sur a norte, y, aunque esta era una ciclorruta muy transitada porque conecta dos importantes universidades con varias zonas residenciales, existía la limitante de llegar al centro de la ciudad en bicicleta, pues se se hacía muy incómodo y riesgoso desde esta zona de la ciudad, siendo uno de los ejes más directos.

Fue así como se realizó un estudio previo a la implementación de la ciclorruta y se identificó que sí existía un frecuente tráfico de ciclistas de oriente a occidente y de occidente a oriente. El estudio mostró que, más o menos, 100 ciclistas se movían por este corredor cada hora, sin embargo, lo que más sobresalía en el análisis era que en esta intersección se registraban un promedio de 78 incidentes viales anuales, de los cuales quedaban 27 personas heridas por año.

La ciudad decidió implementar lo que se llamó una "ruta segura"3, que era una conexión entre oriente-occidente al lado del separador central de la calle San Juan de 3 metros de ancho, reemplazando un carril de carros por dos de bicicletas.

\footnotetext{
${ }^{3}$ Proyecto urbano realizado dentro del marco del Foro Urbano Mundial, en un convenio con ONU Hábitat y la Fundación Despacio con el apoyo del Área Metropolitana, la Alcaldía de Medellín y, principalmente, la comunidad.
} 


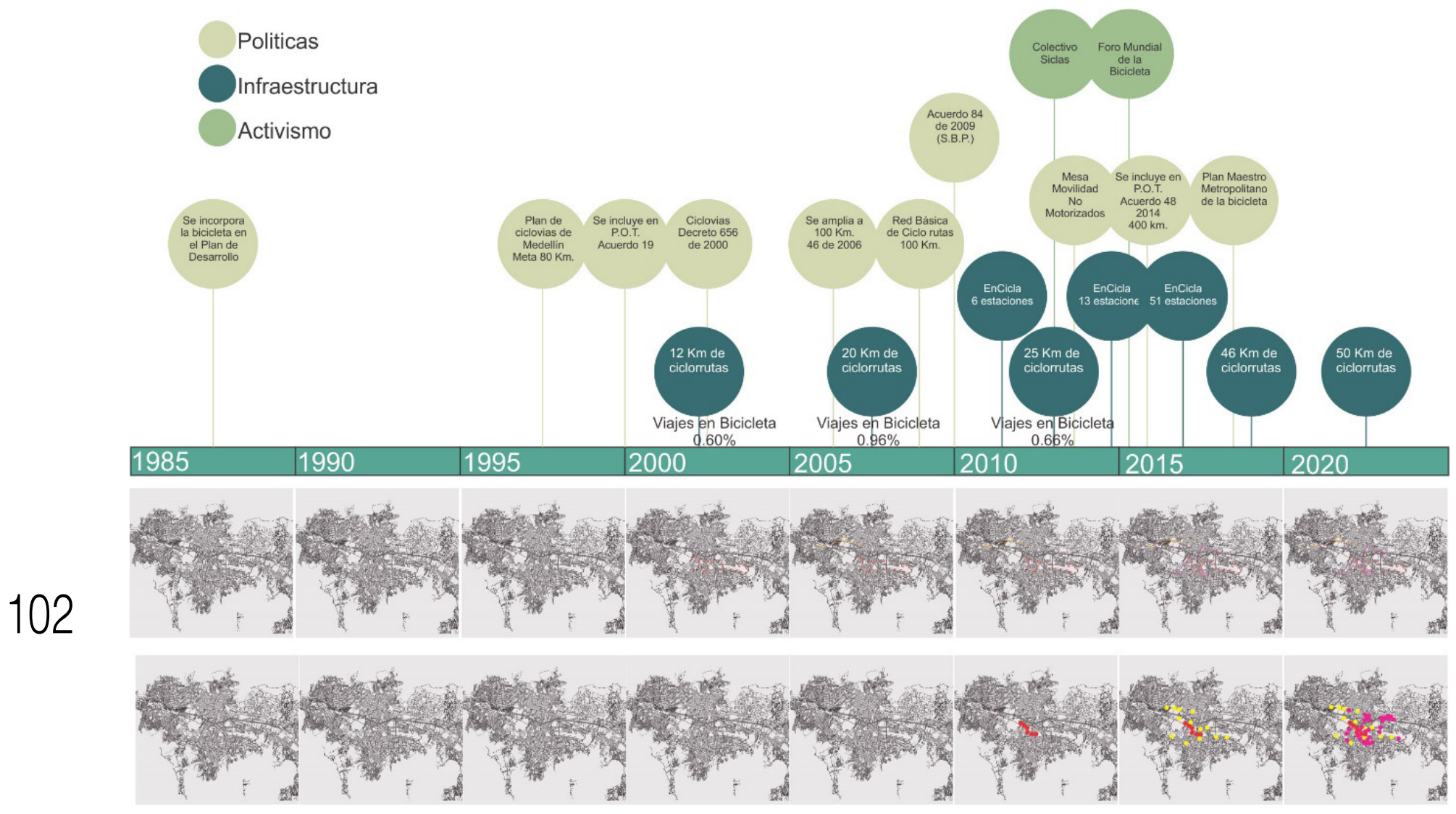

Fig. 22: Evolución de la ciclorruta de San Juan dentro del contexto local.

Fuente: elaboración propia.

Como resultado, en menos de un año, se duplicó la cantidad de ciclistas que se movían por ese corredor y, lo más importante, se redujeron los incidentes viales en un $21 \%$ y los heridos pasaron de 27 a 21 personas ${ }^{4}$.

${ }^{4}$ Datos recolectados por Más Urbano entre el año 2014 y 2017.
Para el año 2017 ya se había cuadriplicado las personas que pasaban por ese lugar en bicicleta, lo que le deja a la ciudad cifras muy positivas en términos ambientales, sociales y económicos. Además, se logró llegar a casi la mitad de las personas que se movían por hora en carro en un tercio del espacio. 


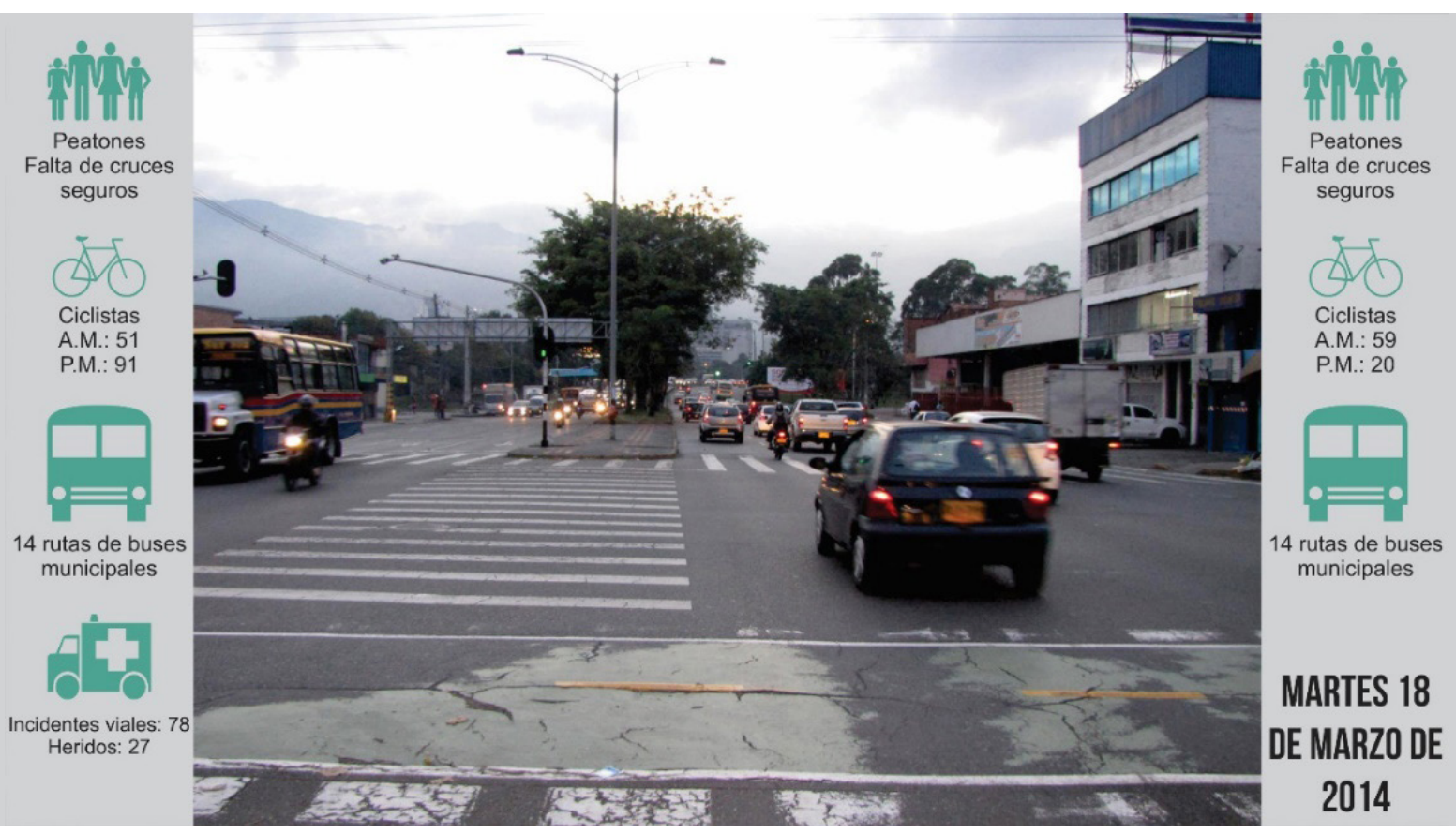

Fig. 23: Análisis calle San Juan - Marzo, 2014.

Fuente: elaboración propia.

A raíz del éxito comprobado, se autorizó la construcción de una ciclorruta definitiva en este lugar. Sin embargo, el municipio no autorizó la ciclorruta en calzada, sino que debía ser desplazada al separador vial existente. Un espacio poco reducido para la cantidad de ciclistas que pasaban por ahí y que seguían creciendo. En consecuencia, para marzo de 2016, es decir 2 años después, se tenía lista la ciclorruta y la cantidad de ciclistas paso de 577 a 1072 en hora pico. No obstante, aunque los incidentes viales seguían siendo los mismos, el hecho de desplazar la ciclorruta al separador provocó que los heridos aumentaran de 21 a 39, demostrando que la ciclorruta en calzada hacia más segura la calle para todos.

Además, se observó también una gran cantidad de personas caminado por el lugar yendo en todas las direcciones, y, por el crecimiento de la cantidad de ciclistas, se les hace cada vez más difícil caminar. Igualmente, se percibió que, aunque muchos ciclistas son respetuosos con las normas y van por el lugar que se supone deben ir, han comenzado a descubrir que pueden hacer otras maniobras y que con estas pueden ahorrar tiempo cruzando por otros lugares, aprovechando los semáforos. 


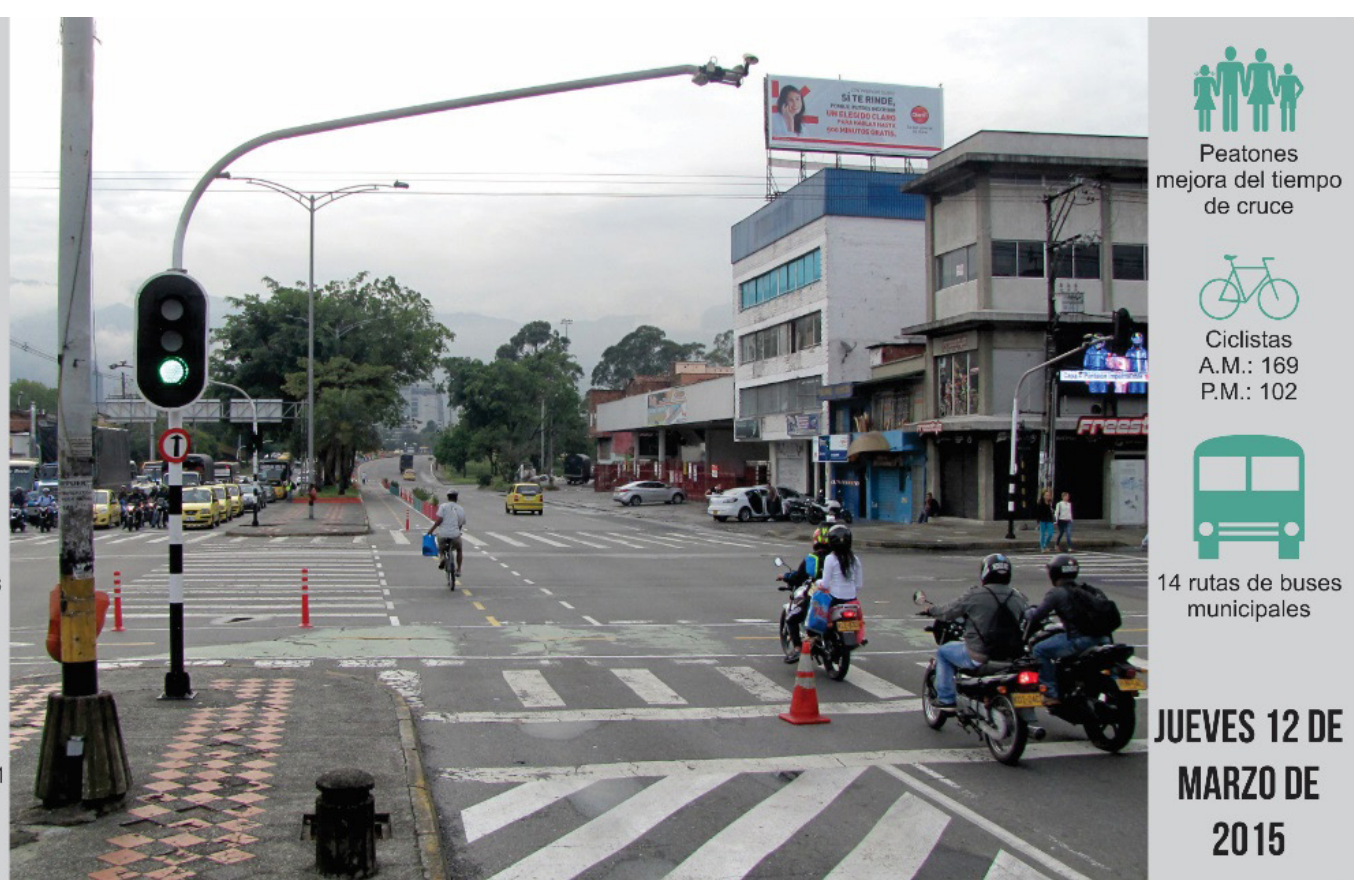

Fig. 24: Movilidad en calle San Juan, año 2015.

Fuente: elaboración Más Urbano.

Esto ha generado algunos conflictos entre ciclistas, peatones y vehículos motorizados.

Precisamente por esto, vale la pena preguntarse: ¿cómo podría ser mejor la ciclorruta en vistas de hacer una calle segura para todos? Se analizaron algunas posibilidades y se encontró que, si se ponía una ciclorruta unidireccional en cada uno de los costados y se ubicaba un carril exclusivo para los buses en la mitad, se podría ayudar a mejorar la movilidad para todas las personas, no solo para los ciclistas sino para todos los que se mueven por el lugar y, así, poder reducir los incidentes viales que incrementan los heridos o muertos.

Si bien esta es solo una propuesta y se necesitarían muchas discusiones para poder llegar a la mejor solución posible para todos, creemos que esta ciclorruta debe empezar a re-discutirse y adecuarse, ya que su impacto en la ciudad es inigualable. Así, la calle de San Juan, con una ciclorruta que fue y debe ser discutida y construida por la ciudadanía, también podría incluir nuevos servicios y calidades para mejorar esta arteria principal de la ciudad. 


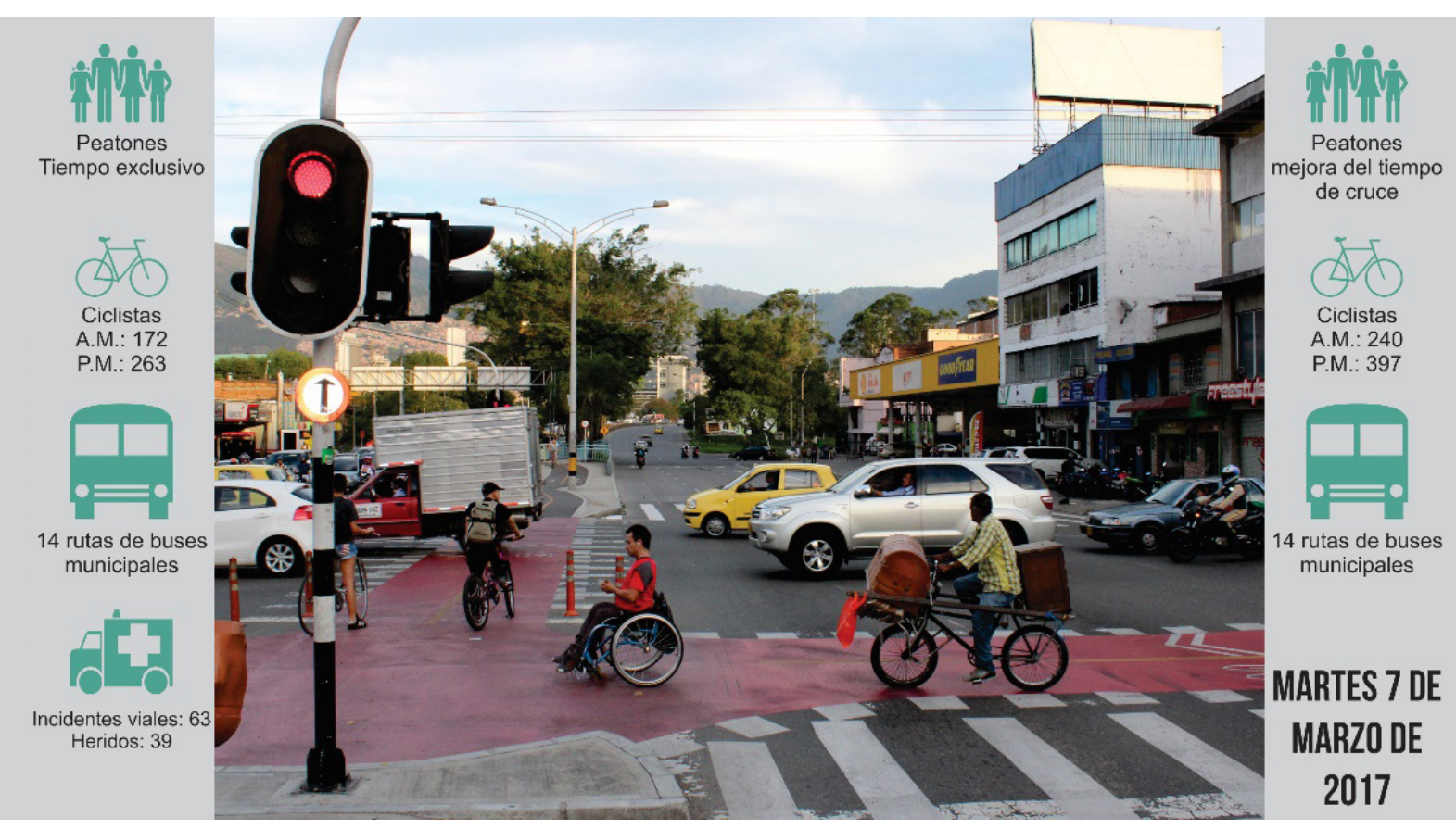

Fig. 25: Movilidad en calle San Juan, año 2017.

Fuente: elaboración Más Urbano.

\section{CONCLUSIÓN}

Pensar en calles para todos o en calles completas no es hablar solo de un proyecto de transformación puntual y rígida, sino en una política de diseño urbano sostenible que da la posibilidad de expandir la calidad en todos los rincones de la ciudad y responder adecuadamente a cada contexto. Ahora bien, dado que existen muchos factores a tener en cuenta al proyectar nuestras calles, debe pensarse en una colaboración interdisci- plinaria que reúna expertos que impulsen los mismos principios. Un proyecto común entre arquitectos, urbanistas, economistas, psicólogos, etc., así como a la comunidad o colectivos sociales, fomentando un funcionamiento integral e inclusivo.

Estos diseños pueden proponer, entre otras opciones, la modificación de un cruce para hacerlo más seguro, la transformación de una calle principal para permitir otro modo de transporte, la implementación de otro tipo de actividad cambiando la amplitud de una vía, 


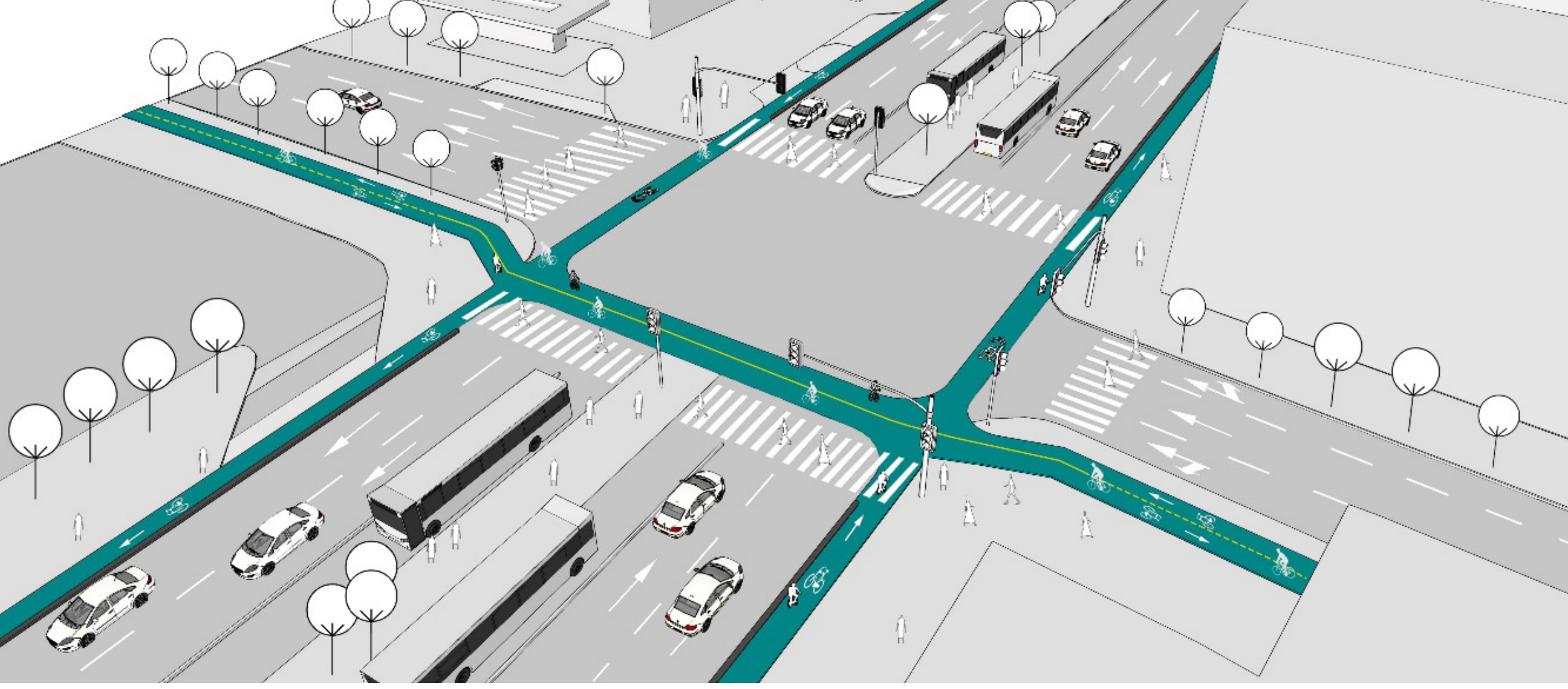

Fig. 26. Propuesta nueva ciclorruta para San Juan.

Fuente: elaboración Más Urbano.

la regulación de la velocidad y horarios de transportes en las calles o la peatonalización de estas; todo esto con el fin de disfrutar de calles que ofrecen ambientes más atractivos, seguros, equilibrados, saludables y eficientes.

Asimismo, se genera un continuo en el espacio público de forma sistémica que, no solo longitudinal sino también transversalmente, enlaza y atrae a su paso; un elemento potencial de la cohesión urbana multiescala que ofrece a las personas una variedad de opciones para moverse.
Finalmente, guardando como propósito la satisfacción del usuario, habría también que procurar saber aún más sobre la percepción y necesidades de los habitantes. De hecho, puesto que la vida urbana se empieza a crear por la escala más pequeña, más humana, el usuario tiene que figurar a la vez como principio y final del proyecto. De este modo, se devuelve el espacio público a la comunidad dándole a la participación ciudadana toda su función democrática, ya que, si las calles son para todos, nuestras ciudades serán para todos. 


\section{REFERENCIAS}

AA.VV. (2014). El atlas de las metrópolis. (F. Mondiplo, Ed.) Valencia: Le monde diplomatique en español-UNED.

Área Métropolitana del Valle del Aburrá. (2017). Encuesta de Movilidad Origen y Destino. Recuperado en mayo de 2018, de http://www.metropol.gov.co/ encuesta_od2017_v2/index.htm|\#/

Busquets, J. (2004). Barcelona. La construcción urbanística de la ciudad compacta. Barcelona: Ediciones del Serbal.

Camarasa, V. (2015). El Plan Haussmann en París. Geobiombo. Obtenido de: https://vicentecamarasa. wordpress.com/2015/03/22/el-planhaussmann-en-paris/

Cerdá, I. y Soria i Puig, A. (1996-[1867]). Cerdá. Las cinco bases de la teoría general de la urbanización. (A. Soria y Puig, Ed.) Madrid: Electa.

Colville-Andersen, M. (11 de septiembre de 2014). The Arrogance of SpaceParis, Calgary, Tokio. Copenhagenize. [Blog]. Obtenido de http://www.copenhagenize.com/ search?q=arrogance + of + space

Dane. (2005-2020). Proyecciones de Población - DANE. Recuperado de www.dane.gov.co\%2Ffiles\%2Finvestigaciones\%2Fpoblacion\%2Fproy epobla06_20\%2FProyeccionMunici pios2005_2020.xls\&usg=AOvVaw0 glBFWYNOCxnfisNpqwnq0
Gehl, J. (2009). La humanización del espacio urbano (5 ${ }^{\mathrm{a}}$ ed.). (M. T. Valcarce, Trad.) Barcelona, España: Editorial Reverté, S.A. (Original en danés, 1971).

Herce, M. y Francesc, M. (2002a). El soporte infraestructural de la ciudad. Barcelona: Ediciones UPC.

Herce, M. y Francesc, M. (2002b). La ingenieria en la evolución de la urbanística. Barcelona: Edicions UPC.

Instituto Nacional de Medicina Legal y Ciencias Forenses. Grupo Centro de Referencia Nacional sobre Violencia. (2017). Forensis 2016. Datos para la vida. Bogotá: Imprenta Nacional.

Portas, N. (2007). A cidade como arquitectura. Lisboa: Livros Horizonte.

Smart Growth America. (Marzo de 2015). Introduction to Complete Streets. Obtenido de https://smartgrowthamerica.org/resources/introductionto-complete-streets/

Smart Growth America. (11 de marzo de 2018). What are Complete Streets? Obtenido de: https://smartgrowthamerica.org/program/nationalcomplete-streets-coalition/what-arecomplete-streets/ 
